\title{
Unsteady Fluid-Structure-Jet Interactions of Agile High-Speed Vehicles
}

\author{
Ryan C. Kitson* and Carlos E. S. Cesnik ${ }^{\dagger}$ \\ University of Michigan, Ann Arbor, MI, 48109, USA
}

\begin{abstract}
This work provides insight to the challenges that should be considered for using attitude control jets with a slender high-speed vehicle and a path forward to modeling the unsteady interaction. At supersonic flow conditions the jet flow interacts with the external flow and induces a complex pressure distribution on the vehicle surface. This interaction has been well documented in the literature for jets mounted on rigid structures and considering steady-state flow. However, the fluid-structure interaction must be considered for a slender vehicle due to increased flexibility. Static and dynamic aeroelastic analysis using the aeroelastic capability of the FUN3D CFD code is presented. The results show that dynamic rigid-body motion and structural deformation have a significant effect on the applied loads. A modeling approach for the unsteady loads is compared against the dynamic aeroelastic solution and the results show good correlation with the CFD solution.
\end{abstract}

\section{Nomenclature}

AoA Angle of Attack, deg

$A_{\text {ref }}$ Vehicle reference area, $\mathrm{m}^{2}$

$a_{\text {ref }} \quad$ Reference speed of sound

$C_{j} \quad$ Force coefficient due to the jet thrust

$C_{j i} \quad$ Force coefficient due to the jet interaction

$C_{m y} \quad$ Pitch moment coefficient

$C_{x} \quad$ Axial force coefficient

$C_{z} \quad$ Normal force coefficient

$f_{c} \quad$ Nonlinear correction factor

$F_{a} \quad$ Force due to the aerodynamics without jet interaction, N

$F_{f s j i} \quad$ Force due to the fluid-structure-jet interaction, N

$F_{j} \quad$ Force due to the jet thrust, N

$F_{j i} \quad$ Force due to the jet interaction, N

$F_{\text {total }}$ Total force applied to the vehicle, $\mathrm{N}$

$F^{u} \quad$ Force applied to the undeformed vehicle, N

$H \quad$ Step response

$h \quad$ Normalized grid spacing

$k \quad$ Step response time index, $1 \ldots n$

$K_{f} \quad$ Amplification factor due to jet interaction on a rigid vehicle

$K_{f}^{f s j i}$ Amplification factor due to fluid-structure-jet interaction

$L_{r e f} \quad$ Vehicle reference length, $\mathrm{m}$

$P R \quad$ Jet total pressure to free-stream static pressure ratio

$t \quad$ Nondimensional time

$\hat{t} \quad$ Time, s

$u \quad$ Model input

$\hat{y} \quad$ Approximate model output

*Ph.D. Candidate, Department of Aerospace Engineering, kitson@umich.edu; AIAA Student Member

†Professor, Department of Aerospace Engineering, cesnik@umich.edu; AIAA Fellow 
y Model output

$y_{\text {linear }}^{\text {ss }}$ Linearized model steady-state output

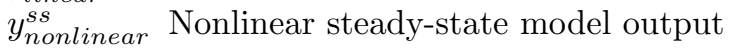

Symbols

$\delta \quad$ Offset term of the nonlinear correction factor

$\eta_{1} \quad$ Lateral First-Bending Mode Deformation, \% tip displacement

\section{Introduction}

TTITUDE control jets have been previously studied as effective control actuators for flight vehicles due to Atheir control authority at very high altitudes, ${ }^{1}$ high angles of attack, and severe aerodynamic heating ${ }^{2}$ compared to conventional control surfaces. In vacuum, the applied force from the jet is equivalent to the thrust, but within the atmosphere there is an interaction between the jet and the free-stream flow. This problem is known in the literature as jet-in-cross-flow or jet interaction (JI) and occurs beyond flight vehicles experiencing the previously mentioned flight conditions. The JI problem has been identified in a range of applications and summarized by Margason ${ }^{3}$ such as smokestacks in cross-wind, pipe flows into rivers, engine fuel injectors, and vertical/short take-off and landing (V/STOL) vehicles. In addition, reaction control systems have been used to control the attitude of hypersonic and supersonic vehicles as seen on the Apollo vehicles, ${ }^{4}$ during aero-braking of the Mars Odyssey, ${ }^{5}$ and expected in future NASA crew exploration vehicles. $^{6}$ The majority of JI analysis has focused on steady-state flow and jet conditions without much attention to the dynamic aspect of the problem. However, additional considerations are required for the vehicle control applications such as the intermittent pulsing of the jet, the vehicle attitude, and vehicle dynamics that all affect the flow and resulting JI.

Roger $^{7}$ presented a summary of fifty years of JI research for flight vehicles that included some key factors that affect JI such as different flow, jet and structural conditions that are all assumed to be constant in time during the experiment. Another important issue that is noted by Roger" is the "jet-on jet-off transients issue" as the steady-state JI develops. Cassel $^{8}$ later summarized the JI with supersonic cross-flow applications and the rise of computational fluid dynamics (CFD) to model and analyze the problem along with experiments and flight testing. However, neither of these review papers focus on the time-domain solution of the problem. For a maneuvering flight vehicle, the flow conditions are inherently dynamic along with the structural dynamics and control inputs. Therefore the time-domain solution of the supersonic JI problem must be considered.

Some experimental and computational work has focused on the transient issue of firing a jet into a supersonic cross-flow. Randolph, Chew, and Johari ${ }^{9}$ fired a pulsating jet into a cross-flow and noted a significant difference in the penetration of the jet into the cross-flow to improve mixing in combustion applications. Experimental results by Naumann et al. ${ }^{10}$ show significant oscillation in the applied forces due to the JI effect. Computational work by Ebrahimi ${ }^{11}$ presents the oscillation and variation of the resultant JI forces and moments due to turning the jet on and off with a characteristic time to develop the steady state on the order of milliseconds. Large Eddy Simulation (LES) of the supersonic JI problem by Génin and Menon ${ }^{12}$ show unsteadiness in the flow field itself with large-scale vortices from the windward side of the jet leading to significant deviations from the time-averaged flow. However, DeSpirito ${ }^{13}$ uses several turbulence models with the Reynolds-averaged Navier-Stokes equations to obtain very good agreement with experimental steadystate results, but notes that the most accurate turbulence model varies with the test conditions. DeSpirito ${ }^{14}$ then examines the transient behavior of the JI and the results show that the integrated forces reach the steady-state value before the pressure distribution. This effect can be neglected for rigid flight vehicles, but the generalized aerodynamic forces of a flexible vehicle are dependent on the pressure and shear force distribution.

Computational work by Sahu, Fresconi, and Heavey ${ }^{15}$ demonstrates the effect of the transient jet forces on the trajectory of a supersonic projectile. Flight simulations of a rigid projectile with control jets were completed with a coupled CFD and rigid-body dynamics model. In this work the total angle of attack was below 20 degrees at Mach 2, the pulsed time was short (2-20 ms) and the total flight time was limited to one second. The jet was fired once with varying initial angular rates and showed the control authority to divert the projectile from its path. The authors concluded that the JI had a significant effect on the projectile trajectory and further study would be required to ensure the accuracy of the projectile. Prince, Chavis

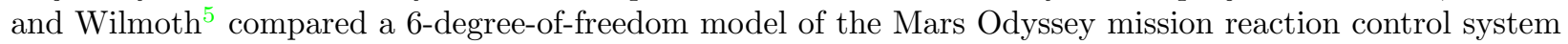
with an aerodynamic interference model to the recorded flight data. The results showed that within the 
atmosphere the aerodynamic interference model was able to capture the vehicle response better than the model without aerodynamic interference, but the amplitude of the body roll rate was off for both approaches. The difference was minimized by using a correction factor that increased the moment due to the interference, which effectively reduced the total thrust effect. The aerodynamic interference model was based on a series of steady-state solutions, which would not capture any of the dynamic aspects during flight simulations. Kitson and Cesnik ${ }^{16,17}$ have developed a flexible high speed vehicle model with a reaction control system to maneuver the vehicle during flight simulations within a coupled modeling and simulation framework. However, the JI model was based on steady-state solutions that do not account for the dynamic flight environment or the transient forces due to varying jet pressure. The effect of dynamic fluid, structure, and jet conditions needs to be considered prior to developing an unsteady JI model to include into a simulation framework.

The previous work in the literature shows the development of the experimental and computational methods to study steady-state JI with a supersonic cross-flow. In addition, previous work has identified the transient JI due to turning the jet on and off and its effect on the vehicle surface pressure distribution. However, JI is not well understood in a dynamic environment that maneuverable vehicles typically operate in. Very high angles of incidence, high angular rates, and structural deformation in particular are conditions that must be considered for slender high-speed maneuverable vehicles.

This paper considers the fluid-structure-jet interaction (FSJI) of a slender high-speed maneuverable vehicle operating in a dynamic environment with reaction control jets for vehicle control. The FUN3D CFD $\operatorname{code}^{18}$ is used for the fluid dynamics and aeroelastic analysis with a previously developed slender high-speed vehicle model. ${ }^{16}$ First, the effect of high angles of attack and structural deformation on JI is assessed. Next, time-accurate simulations of prescribed rigid-body motion and structural deformation at varying frequencies are presented that highlight the dynamic effect on jet interaction. Finally, the vehicle response to the transient jet-on problem is presented using a rigid and flexible vehicle. For each of the time-accurate CFD simulations there is a comparison to an unsteady modeling method to assess the applicability of the method and highlight the nonlinearity of the CFD solution. This work helps characterize the FSJI problem for a maneuvering flexible vehicle to highlight the potential challenges and advantages to using reaction control jets with a slender vehicle.

\section{Numerical Approach}

This section details the approach to the fluid-structure-jet interaction problem of a flexible high-speed vehicle in a dynamic flight environment. First, the vehicle model is presented that is used throughout the analysis. The computational fluid domain is developed for this vehicle to capture the jet interaction and boundary layer effects. Finally, details on the step response and linear convolution analysis is presented that is used to analyze the vehicle responses to changes in flight, jet and structural conditions.

\section{A. High-Speed Vehicle Model}

The vehicle used for this study was developed by the authors ${ }^{16}$ for flight simulations of a flexible high-speed vehicle. The basic dimensions and flight conditions for the vehicle are shown in Table 1. The baseline configuration in the study has a uniform mass and stiffness distribution, therefore the lower frequencies free-free vibration mode shapes are similar to the ones of a uniform free-free beam. The outer mold line of the model, shown in Figure 1, was chosen to move the center of pressure aft of the center of gravity based on the lack of fin surfaces that are conventionally located at the tail end of the vehicle. Markers for the center of pressure (C.P.) and center of gravity (C.G.) have been added to the figure. Conventional fin surfaces are not included in this model due to their loss of effectiveness at high angles of attack. Instead, reaction control jets are located at $25 \%$ of the vehicle length normal to the body surface to control the vehicle orientation.

The free-free vibration modes of the vehicle were used with the aeroelastic module of FUN3D and specifically during the dynamic FSJI analysis. The first three bending modes of the vehicle are used during the simulation with the first mode being the dominant mode of deformation. The first mode shape is also prescribed as a function of time for the forced structural vibration of the vehicle. All three of the structural modes are presented in Figure 2. 
Table 1. Basic properties and flight conditions for the vehicle

\begin{tabular}{lrl}
\hline \hline Property & Value & Unit \\
\hline Total mass (uniform distribution) & 85 & $\mathrm{~kg}$ \\
Length, $L_{\text {ref }}$ & 3 & $\mathrm{~m}$ \\
Reference Area, $A_{\text {ref }}$ & 0.195 & $\mathrm{~m}^{2}$ \\
Diameter at mid-length & 0.13 & $\mathrm{~m}$ \\
Mach & $2-4$ & \\
Altitude & $12-30$ & $\mathrm{~km}$ \\
\hline
\end{tabular}

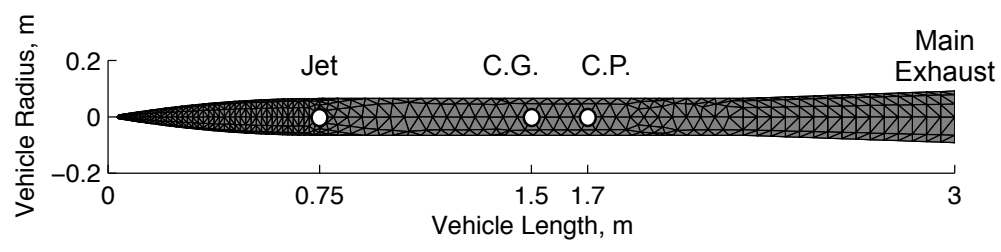

Figure 1. Side view of the undeformed axisymmetric vehicle ve $^{16}$
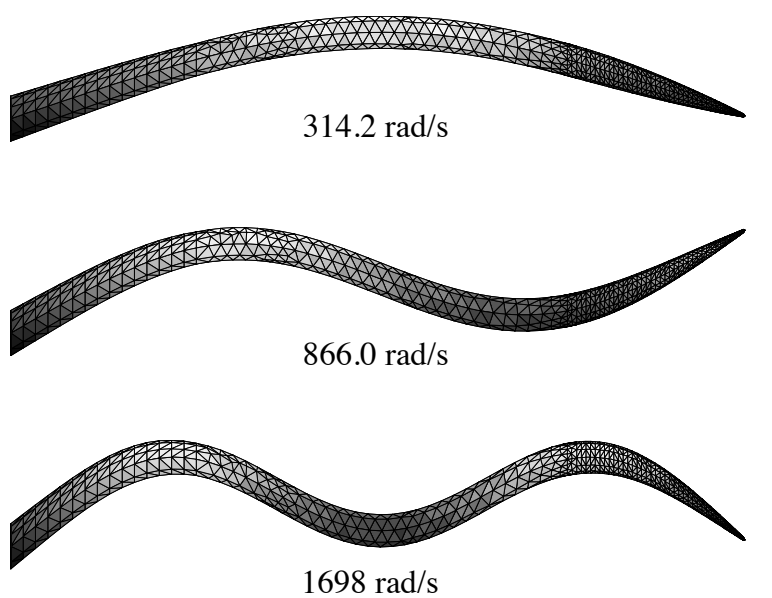

Figure 2. Free-free vibration mode shapes and corresponding frequencies of the vehicle with uniform mass and stiffness ${ }^{16}$

\section{B. Jet Interaction Computational Modeling}

This section describes the CFD modeling and analysis of a representative high speed vehicle. The discretization of the computational domain was done using Pointwise and used with the NASA FUN3D CFD code. ${ }^{18}$ The code was used to solve the Reynolds-averaged Navier-Stokes equations with the Spalart-Allmaras (SA) turbulence model for the vehicle at various flow conditions. FUN3D also has a built-in aeroelastic component that allows the user to prescribe surface motion at each time step or provide surface mode shapes. The surface mode shapes are used to solve the linear structural equations for dynamic aeroelastic analysis.

A grid convergence study was completed that uses a full three-dimensional grid and these results are presented in Figure 3. The grid spacing values are listed in Table 2 as well as the conditions used for the study in Table 3. The farfield domain is an ellipsoid located $L_{r e f}$ away from the vehicle surface. The undeformed vehicle at a nonzero angle of incidence with the attitude jet on is used to create an asymmetric condition for the jet interaction. Surface slices were chosen to highlight the convergence of the jet interaction profile as shown in Figure 3(a)-(b). The lengthwise slice coordinate is normalized by $L_{r e f}$ and the spanwise 
coordinate is normalized by the diameter of the vehicle. The fine grid with normalized spacing $h$ equal to 1 is used to obtain the numerical results in this study.

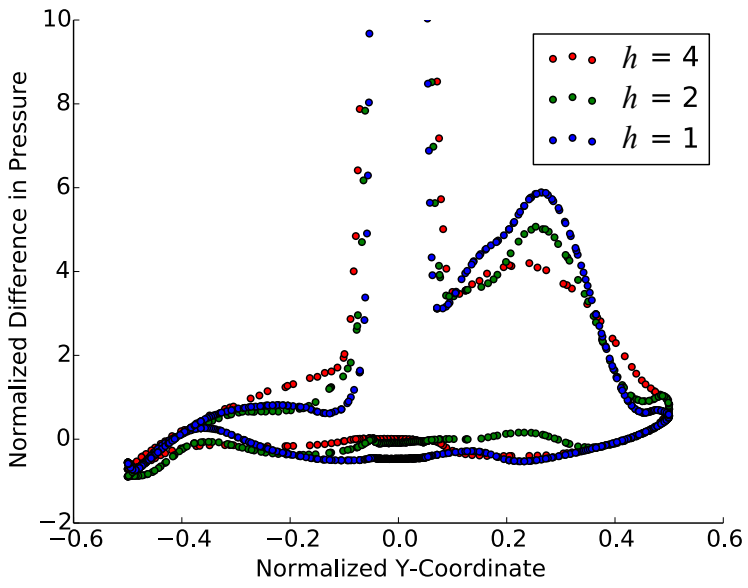

(a) Spanwise Slice Through Jet

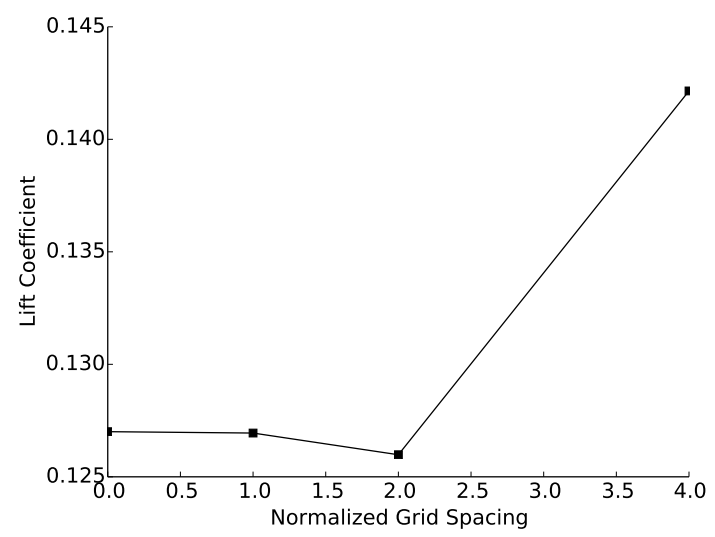

(c)

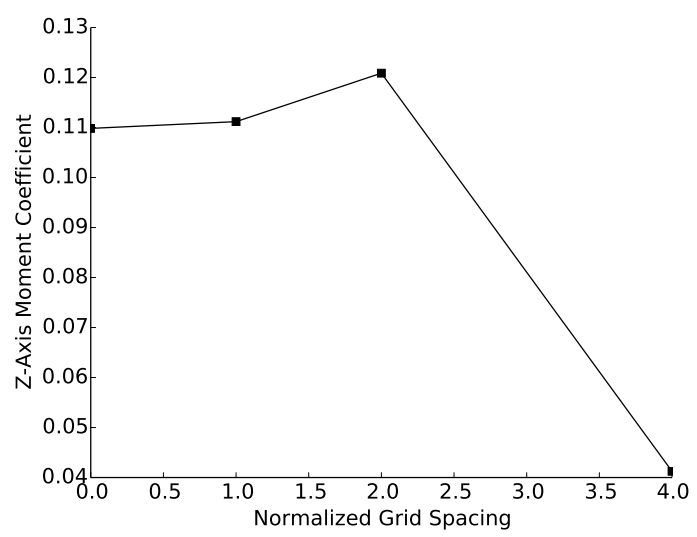

(e)

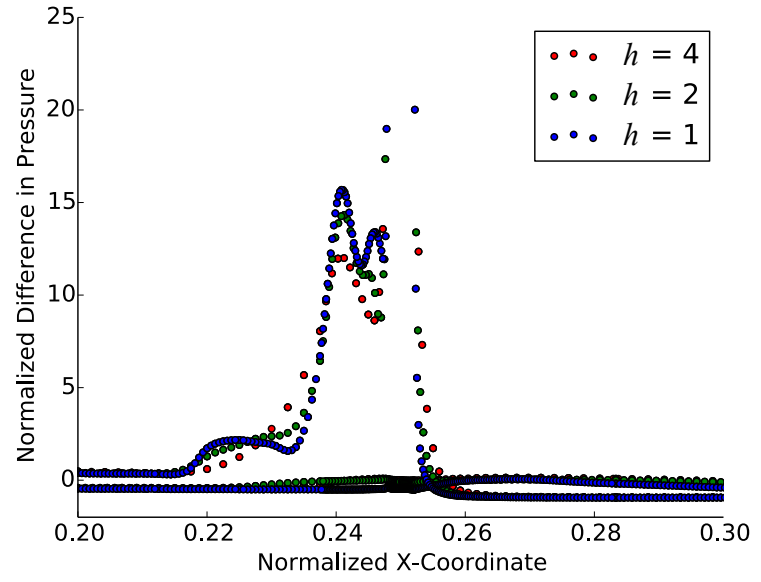

(b) Lengthwise Slice Through Jet

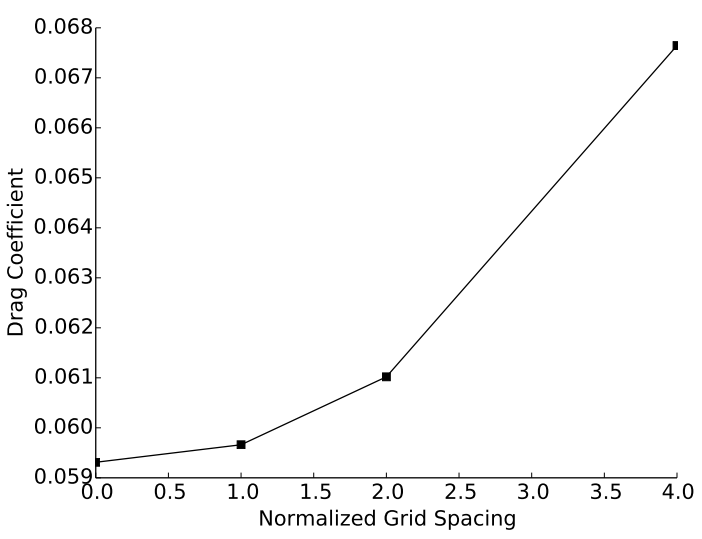

(d)

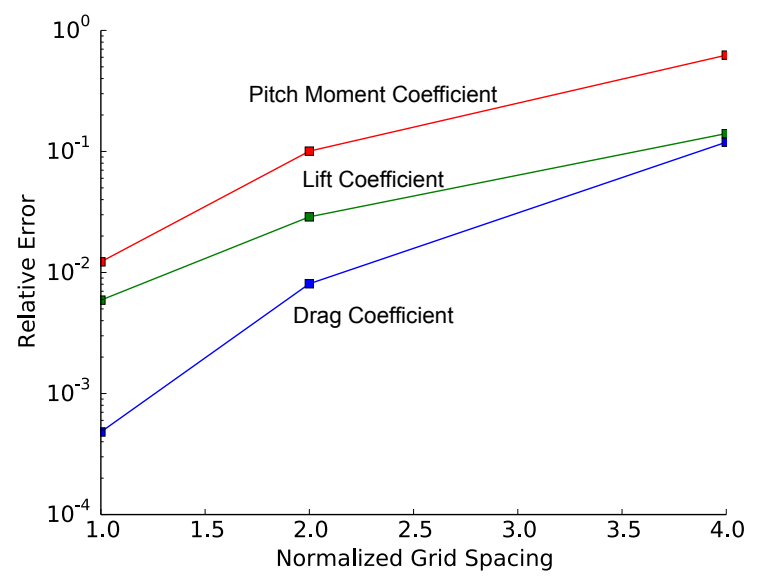

(f)

Figure 3. The surface distribution and integrated coefficients converge with refined grid spacing, $h$. 
Table 2. Grid Spacing for the Grid Convergence Study

\begin{tabular}{cccc}
\hline \hline Grid Spacing & $y+$ & Surface Spacing & Farfield Spacing \\
\hline 4 & 4 & $L_{\text {ref }} / 150$ & $L_{\text {ref }} / 8$ \\
2 & 2 & $L_{\text {ref }} / 300$ & $L_{\text {ref }} / 16$ \\
1 & 1 & $L_{\text {ref }} / 600$ & $L_{\text {ref }} / 32$ \\
\hline
\end{tabular}

Table 3. Conditions for the Grid Convergence Study

\begin{tabular}{lll}
\hline \hline Parameter & Value & Units \\
\hline Length Reynolds Number & $3.49 \times 10^{7}$ & $1 / \mathrm{m}$ \\
Mach & 3.0 & \\
Angle of Attack & 10 & $\mathrm{deg}$ \\
Angle of Sideslip & 10 & $\mathrm{deg}$ \\
Jet Total Pressure & 17.5 & $\mathrm{MPa}$ \\
Jet Total Temperature & 2700 & $\mathrm{~K}$ \\
Jet Exit Mach Number & 1 &
\end{tabular}

\section{Jet Interaction Amplification Factors}

This section presents the jet interaction amplification factor to measure the ratio of total force or moment applied to vehicle due to the jet and jet interaction to the force jet vacuum thrust. A value less than 1 is a de-amplification of the control input, less than 0 indicates control reversal, and greater than 1 is an amplification of the control input. For a rigid vehicle this amplification factor $K$ follows the convention in the literature ${ }^{14,19,20}$ defined as,

$$
K_{f}=\frac{F_{j}+F_{j i}}{F_{j}}=\frac{C_{j}+C_{j i}}{C_{j}}
$$

where $K_{f}$ is the force amplification factor, $F_{j}$ the jet thrust, and $F_{j i}$ the force due to jet interaction. $F_{j i}$ is calculated by,

$$
F_{j i}=F_{\text {total }}-F_{a}-F_{j}
$$

where $F_{\text {total }}$ is the total force on the vehicle, $F_{a}$ the force due to aerodynamics only. Normalizing the forces and using the force coefficients $\left(C_{j}, C_{j i}\right)$ results in the same amplification factor.

A new FSJI amplification factor, $K_{f}^{f s j i}$, is introduced to capture the fluid-structure interaction effect in addition to the jet interaction effect. The amplification a quantity $F$ due to FSJI effects is calculated as,

$$
K_{f}^{f s j i}=\frac{F_{f s j i}+F_{j}+F_{a}^{u}}{F_{j}+F_{a}^{u}}
$$

where $F_{f s j i}$ is the difference in the total quantity $F_{t o t a l}$ and the value due to aerodynamic loading over the undeformed structure $F_{a}^{u}$ and jet thrust $F_{j}$, as

$$
F_{f s j i}=F_{t o t a l}-F_{j}-F_{a}^{u}
$$

\section{Linear Unsteady Modeling and Nonlinear Correction}

The vehicle step response can convolved with the input time history to approximate the system output. For a linear system, this would be the exact solution. The implementation used in this paper is based on the work by Raveh and Mavris ${ }^{21}$ that showed how a step response as opposed to an impulse response could be used to calculate unsteady loads. For example, during the vehicle simulation the angle of attack history $u$ is used with the step response $H$ with the discrete version of Duhamel's integral to calculate the aerodynamic load at the current time step $y[n]$ as

$$
y[n]=u[0] H[n]+\sum_{k=1}^{n}(u[k]-u[k-1]) H[n-k]
$$


The first term $u[0] H[n]$ represents the steady aerodynamic load at index 0 while the second term is the unsteady component due to an arbitrary input from index 1 to $n$. Therefore, the steady term can come from a more accurate source such as a steady-state CFD solution and the unsteady contribution of the most recent time steps can be calculated using convolution. The result is a linear approximation of the CFD solution for the arbitrary input.

A steady-state solution from the nonlinear CFD model can be used to correct the approximated unsteady loads using the nonlinear correction factor approach presented by Skujins and Cesnik. ${ }^{22}$ The method is based on the assumption that the ratio of the nonlinear to linear unsteady prediction is approximately equal to the ratio of nonlinear to linear steady prediction. Therefore, at each time step the vehicle state is used to approximate the steady-state solution $y^{s s}$ using the nonlinear CFD surrogate and linear convolution models. The ratio of these steady-state approximations is known as the nonlinear correction factor, $f_{c}$. The corrected unsteady approximation $\hat{y}$ can be written as

$$
\hat{y}=f_{c} y
$$

where $y$ is the linear convolution of step response and input history in Eq. (5), and $f_{c}$ is defined as

$$
f_{c}=\frac{y_{\text {nonlinear }}^{\text {ss }}}{y_{\text {linear }}^{\text {ss }}}
$$

To avoid division by zero, an offset term $\delta$ can be introduced that still preserves the effect of the nonlinear correction factor such as

$$
f_{c}=\frac{y_{\text {nonlinear }}^{\text {ss }}+\delta}{y_{\text {linear }}^{s s}+\delta}
$$

\section{Results}

The numerical results focus on the dynamic aspect of the FSJI problem using high-fidelity CFD. From a modeling perspective the nonlinearity of the system should be understood to select the appropriate method for approximating the dynamic loads due to the FSJI. The unsteady CFD solutions are used to investigate the nonlinearity of the problem by comparing the results to a linearized model. The linearized model is based on the linear convolution of step responses of the vehicle to changes in each of the flow, structure, and jet parameters. These step responses are then convolved according to the time history of inputs to the unsteady CFD solutions to approximate the vehicle loads. The nonlinearity of the system is quantitatively assessed by measuring the difference between the linearized model and the nonlinear unsteady CFD solutions.

One prospective modeling method is assessed against the unsteady CFD solutions by using the linear convolution with nonlinear correction factor approach. The nonlinear correction factor is derived from the steady-state FSJI solutions. At each time step of a dynamic solution the approximate loading from the linear model is corrected by the steady-state value following the approach discussed in Section II. Comparing the linear convolution and nonlinear correction factor method to the unsteady CFD solutions determines the applicability of using this method to approximate the vehicle loads over the whole flight envelope.

In each of the following sections dynamic simulations are presented with respect to the nondimensional time, $t$, calculated as,

$$
t=\hat{t} \frac{a_{r e f}}{L_{r e f}}
$$

where $\hat{t}$ is the dimensional time in seconds, $L_{r e f}$ the vehicle reference length, and $a_{r e f}$ the reference speed of sound.

The body-fixed forces and moments are reported with the axial force $\left(C_{x}\right)$ positive aft, normal force $\left(C_{z}\right)$ positive up, and pitch moment $\left(C_{m y}\right)$ positive nose-up.

\section{A. Jet Interaction Sensitivity}

The jet interaction to changes in the flow and structural conditions is important to characterize prior to studying the unsteady response. In this case the highly maneuverable vehicle has a wide range of operating conditions that should be understood including high angles of attack and moderate structural deformations. Steady-state solutions of the vehicle with jet interaction over a wide range of conditions can be expensive to repeatedly sample to characterize the sensitivity. Quasi-steady maneuvers can provide a better resolution of 
the sensitivity at a lower cost. This quasi-steady approach is taken for studying the sensitivity of the vehicle loads due to aerodynamics and jet interaction over a wide range of angles of attack and vehicle deformation.

The vehicle range of angle of attack is sufficiently high to achieve large normal accelerations. In addition, the attitude jet thrust has a range that is sufficient to counter-act the aerodynamic stiffness of the vehicle leading to very large angles above 90 degrees. However, from previous work by the authors ${ }^{17}$ it is shown that there is a nonlinear transition between the jet interaction with a windward and leeward facing jet, which occurs near zero angle of attack of a rotating vehicle. The dynamic transition between windward and leeward is expected to be more significant and is the subject of the current work compared to the dynamic effects at very high angles of attack. The mean condition of the fluid, structure, and jet parameters about which the sensitivity is studied is listed in Table 4 with the range of the quasi-steady angle of attack sweep in Table 5 .

Table 4. Quasi-Steady Sensitivity Study Mean Conditions

\begin{tabular}{ccc}
\hline \hline Parameter & Value & Units \\
\hline Altitude & 21.3 & $\mathrm{~km}$ \\
Mach & 3.0 & \\
Angle of Attack & 0 & degrees \\
Angle of Sideslip & 0 & degrees \\
Structural Deformation & $0 \%$ & tip deflection \\
Jet Total Pressure & 17.5 & $\mathrm{MPa}$ \\
Jet Total Temperature & 2700 & $\mathrm{~K}$ \\
Jet Exit Mach Number & 1 & \\
Main Exhaust Mass Flow Rate & 0.2 & $\mathrm{~kg} / \mathrm{s}$ \\
Main Exhaust Total Temperature & 2700 & $\mathrm{~K}$ \\
Main Exhaust Exit Mach Number & 3 & \\
\hline
\end{tabular}

Table 5. Quasi-Steady Sensitivity Study Sweep Conditions

\begin{tabular}{ccc}
\hline \hline Parameter & Value & Units \\
\hline Angle of Attack & $0-360$ & degrees \\
Structural Deformation & $0-5 \%$ & tip deflection \\
\hline
\end{tabular}

The results in Figure 4 show the vehicle response with the attitude jet on and off along with the corresponding JI amplification factor $K$. The lift and drag coefficients are normalized using the jet thrust normal to the body while the pitch moment is normalized by the moment due to the jet thrust. The coefficients represent the effect due to aerodynamics and jet interaction without the contribution of the jet thrust. As previously discussed, the amplification factor is significantly different as the vehicle transitions from negative to positive angles of attack. In addition, there is a transition point near 45-50 degrees angle of attack that is a crossover in the sign of amplification factor. At low positive angle of attack the jet interaction amplifies the moment in the intended direction whereas at high positive angle of attack the jet interaction reverses the effect of the jet thrust at these conditions.

The vehicle pressure distribution due to aerodynamic pressure and jet interaction must be understood for moderate deflections of the vehicle. This type of analysis is imperative for a flexible vehicle operating in a high speed condition performing aggressive maneuvers that impose large forces on the structure. However, this type of analysis is largely missing from the literature especially including the effect on the jet interaction pressure distribution. The analysis in this section takes the quasi-steady approach to CFD solutions and imposes a deformation as a function of time while solving for the flow around the vehicle. The mean condition about which the quasi-steady maneuver is performed is provided in Table 4 and the range of structural deformation is listed in Table 5 .

The forces and moments of the vehicle with JI is presented in Figure 5 along with the calculated FSJI amplification factor. The jet interaction acts as an offset to the equivalent values using an undeformed structure and the structural deformation has an additional effect particularly on the applied moment. In 


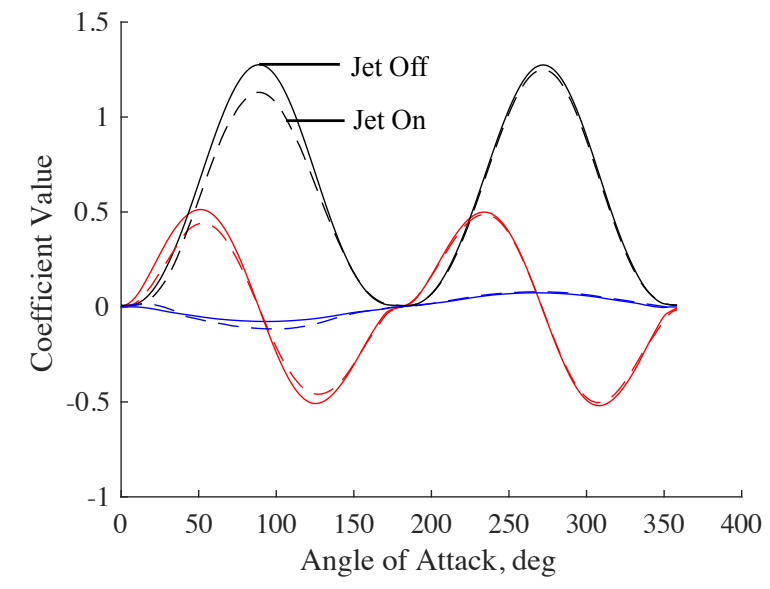

(a) Response with Jet On and Off

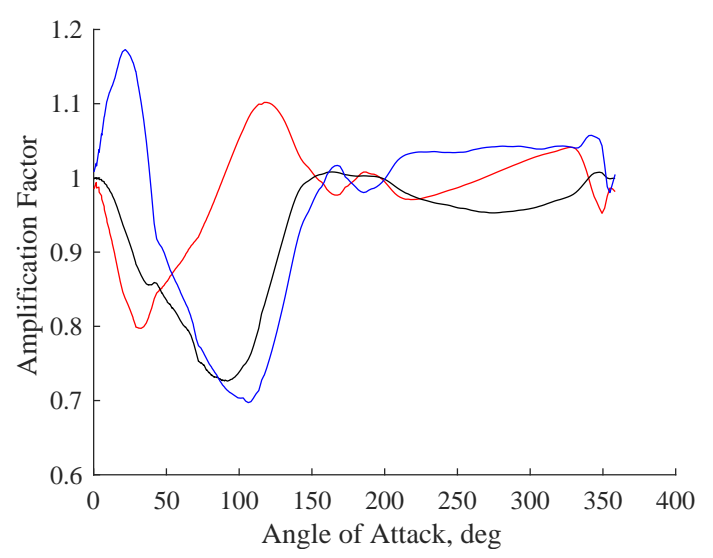

(b) Jet Amplification Factor

Figure 4. Jet Interaction Sensitivity to Large Angles of Attack. Normal Force (red), Axial Force (black), and Pitch Moment (blue)

addition, although the simulation was intended to be quasi-steady there is a slight lag that is seen in the normal force coefficient despite the drag and pitch moment showing a repeating solution. The unsteady model depends on the steady solution for a nonlinear correction and this solution will add a complication to recover the nonlinear unsteady solution. However, it is interesting to note that even at this relatively low frequency the expansion region that extends over a significant portion of the vehicle is time-dependent.

\section{B. Step Responses to Changes in Fluid, Structure, and Jet Conditions}

The vehicle response to step changes in the various parameters is calculated and the results serve two main functions. First, the sensitivity of the applied loads as a function of a dynamic input can be studied. Second, the step response about the mean condition can be used to create a linearized model of the system.

The dynamic effects of the coupled FSJI has not been significantly investigated previously and these results add to the body of work in the fluid-structure and JI research areas. The JI problem has been studied extensively for steady-state problems, but the transient nature of the problem is not extensively present in the literature. The highly maneuverable vehicle under consideration introduces several dynamic phenomena to the problem. The flow conditions are unsteady as the vehicle maneuvers imposing angular rates and accelerations with respect to the free-stream flow direction. The structure deforms and oscillates in response to the unsteady aerodynamic loads and the time-dependent control inputs. Finally, the jet thrust is time-varying as well as the JI with the external flow. The unsteady effects and dynamic response of the FSJI can be initially understood by investigating the response to step changes in each of the fluid, structure, and jet domains.

Some of the over-arching objectives of this work are to analyze, model, and exploit the dynamic FSJI to improve the performance of high-speed vehicles. The step responses can be used to analyze the dynamic FSJI as well as build a linearized model of the dynamic FSJI. The approach is described in Section II to predict the vehicle loads due to time-dependent changes in the fluid, structure, and jet conditions. The step responses will then be combined with the nonlinear sweeps of the angle of attack and structural deformation domains to create a nonlinear correction factor to augment the linearized model.

The angle of attack step response is performed about the mean conditions (Table 4) and a 3.6-degree step input with the jet off. The resulting forces and moments are presented in Figure 6(a) that will be used for the unsteady surrogate model and compared to the nonlinear solution of a rigid pitch maneuver. The step structural deformation is calculated about the mean condition with the jets off and a step in the first free vibration mode shape equal to tip deformation of $1 \%$ vehicle length. The resulting forces and moments are presented in Figure 6(b) that will be used for approximating the unsteady loads of a vibrating vehicle with JI. The transient solution to turning on the jet is investigate using a step input from the off condition to a total pressure of $350 \mathrm{kPa}$ with a rigid vehicle. The results presented in Figure 7 show the jet interaction response time to reach a new steady state is approximately 10-20 times longer than the fluid and structure 


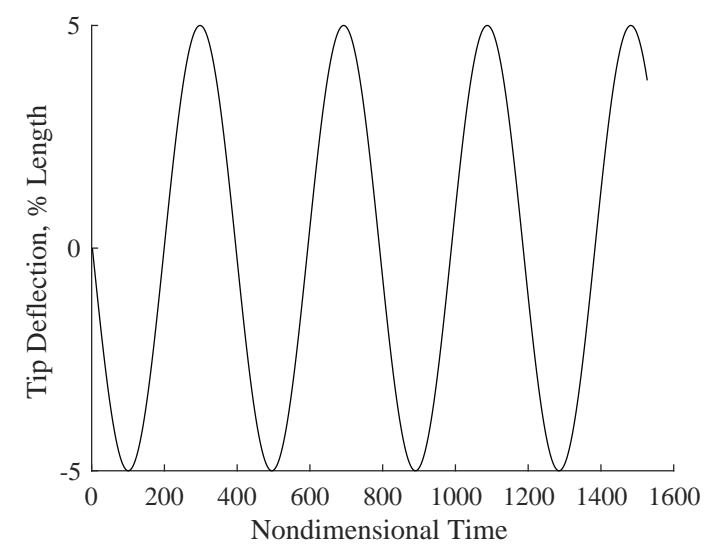

(a) Structural Deformation Input

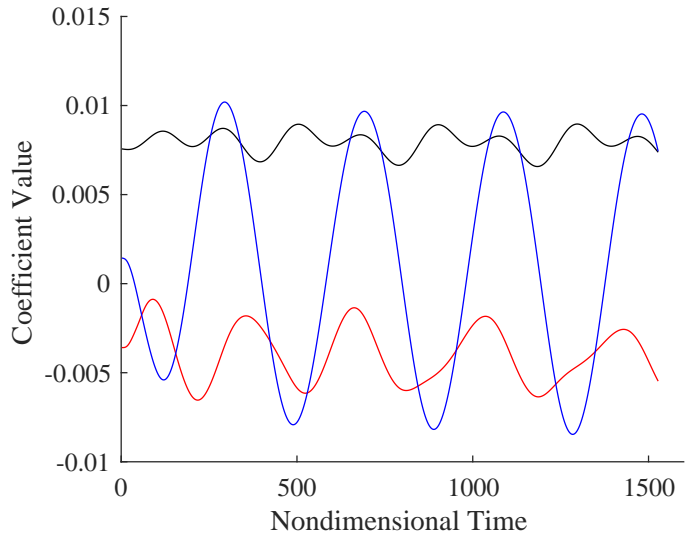

(b) CFD Solution

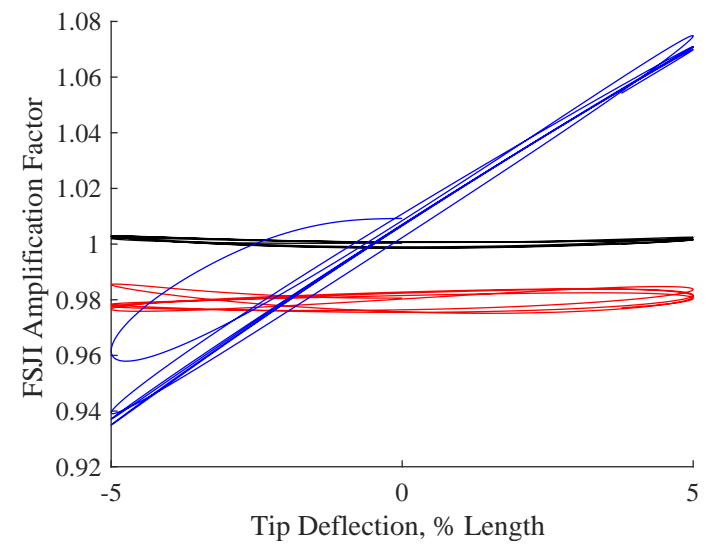

(c) Amplification Factor due to FSJI

Figure 5. Jet Interaction Sensitivity to Moderate Structural Deformation. Normal Force (red), Axial Force (black), and Pitch Moment (blue)

response times.

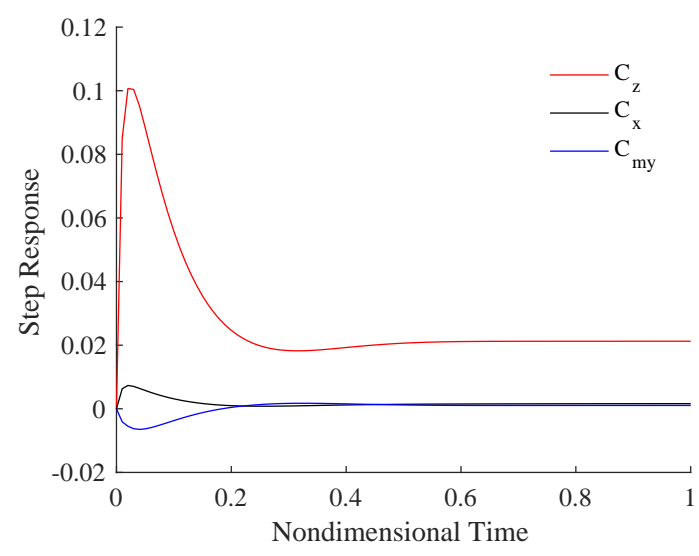

(a) Angle of Attack

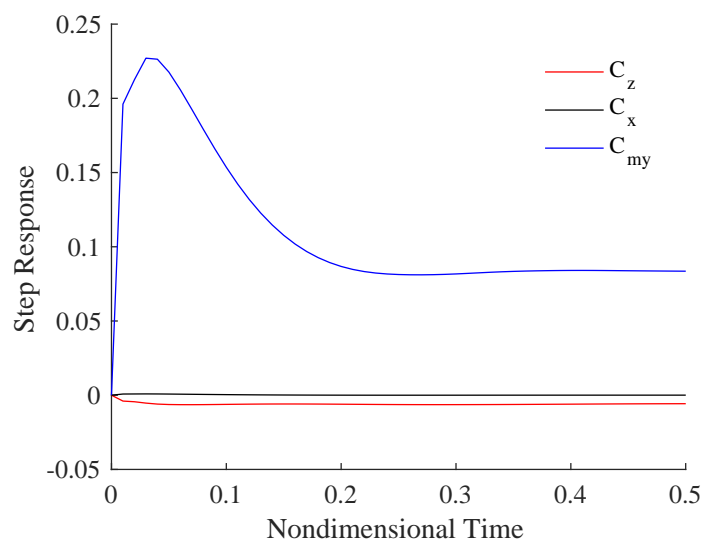

(b) Deformation

Figure 6. Step Responses to 3.6-degree-step Angle of Attack, 1\% first bending mode step deformation inputs. Altitude 17.7 km, Mach 3.0 


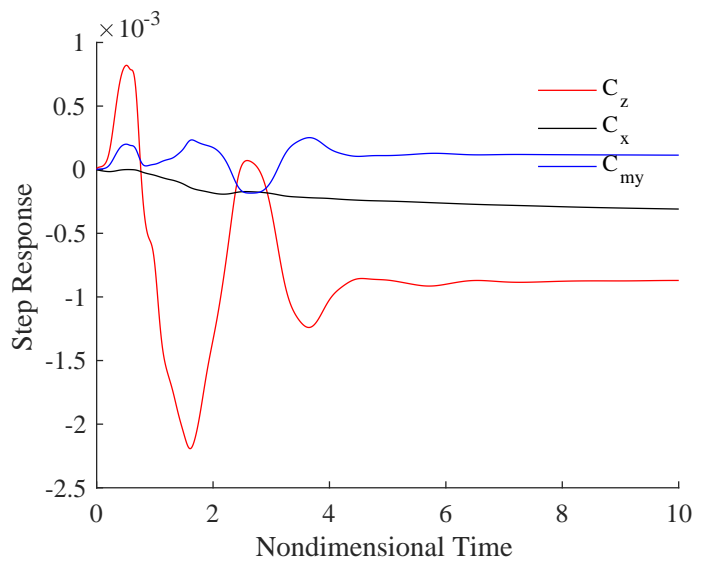

(a) Jet-On

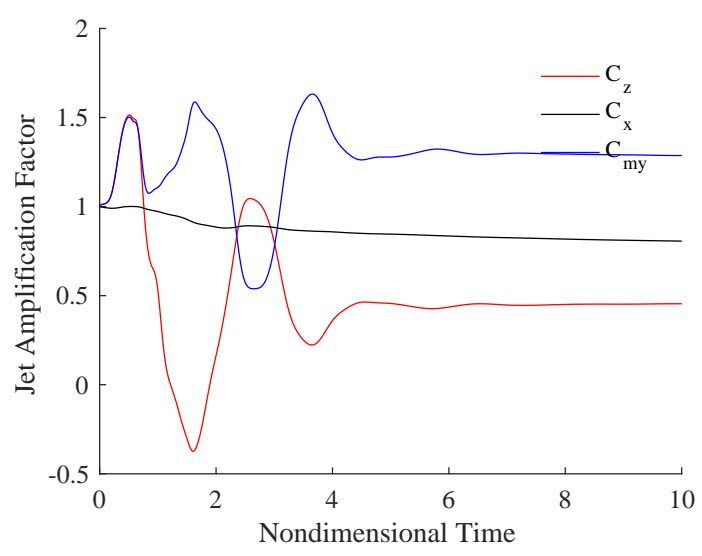

(b) Transient Jet Amplification Factor

Figure 7. Transient Jet-On Response and Amplification Factor to a $350 \mathrm{kPa}$ step in jet total pressure. Altitude $17.7 \mathrm{~km}$, Mach 3.0

\section{Dynamic Fluid-Structure-Jet Interaction Simulations}

Dynamic fluid-structure-jet interaction simulations of the agile high-speed vehicle are presented to understand the dynamic effects and the nonlinearity of the system. Three simulations in particular are chosen to study the dynamic FSJI problem. The first simulation is a forced pitch oscillation of a rigid vehicle with the attitude jet on. This simulation examines the effect of angular rate on the applied loads about the mean angle of attack with varying frequency. The frequency is increased to add unsteadiness in the flow and measure the effect on the applied forces and moments. The second simulation prescribes the structural deformation as a function of time with the jet interaction effect. The first free vibration mode is used with increasing levels of frequency up to the first bending mode free vibration frequency. The third simulation examines the response to transient jets with a flexible vehicle to reveal how the structural degrees of freedom interact with the external and jet flows. The time-accurate fluid-structure capability of FUN3D is used for this analysis to couple the linear structural equations with the nonlinear flow solution.

These nonlinear dynamic simulations can be approximated using the linear convolution of the step responses of the vehicle to the various fluid, structure, and jet parameters. Comparing the linear response to the same conditions as the nonlinear model reveals the feasibility of using a linearized approximation. The linear model is then extended by using the nonlinear steady-state solution of the vehicle, which applies a correction factor to achieve better correlation with the nonlinear response. The linear model with nonlinear correction factor represents one modeling strategy that may be used to model the dynamic FSJI over a full range of operating conditions. Developing the model for a single combination of Mach and altitude examines the feasibility of this modeling approach prior to further model development.

A pitch maneuver is prescribed for which the oscillation frequency is increasing throughout the simulation to study the impact of angular rates on the resultant vehicle loads. The pitch frequency ranges from $1 \mathrm{~Hz}$ to just under $25 \mathrm{~Hz}$, which corresponds to a reduced frequency from 0.01 to 0.27 , with an amplitude of 7.2 degrees and flight conditions listed in Table 6. The history of the input and calculated loads are presented along with the associated amplification factors in Figure 8. As the frequency of oscillation increases there is a gain in the amplification factors due to jet interaction, which demonstrates how the jet interaction is dependent on the steady and unsteady flow conditions. The amplification factors oscillate about the initial value, approximately equal to 1 , but show minimum values below 0 indicating a reversal.

Table 6. Rigid-Body Motion Simulation Conditions

\begin{tabular}{cccc}
\hline \hline Altitude, $\mathrm{km}$ & Mach & $|A o A|$, deg & Jet Total Pressure, $\mathrm{MPa}$ \\
21.3 & 3.0 & 7.2 & 17.5 \\
\hline
\end{tabular}

The unsteady model prediction is presented alongside the nonlinear solution (Figure 8(d)) to highlight the nonlinear dynamic response as a function of angular rate. The steady and unsteady models do not correlate 


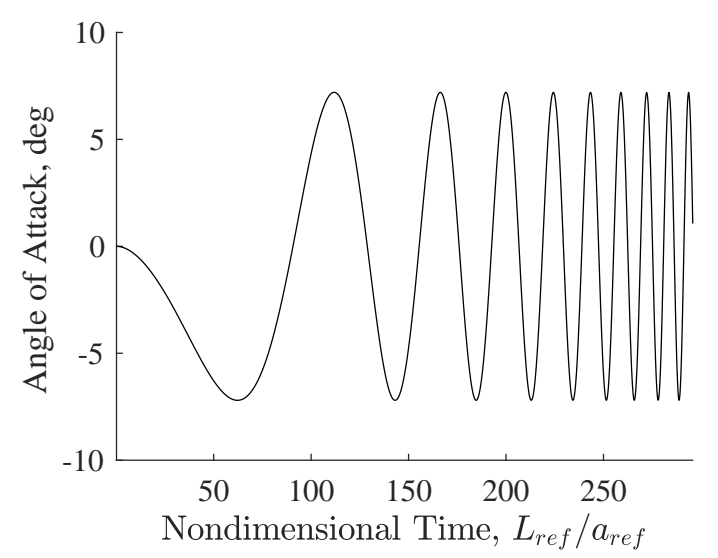

(a) Forced Pitch Input

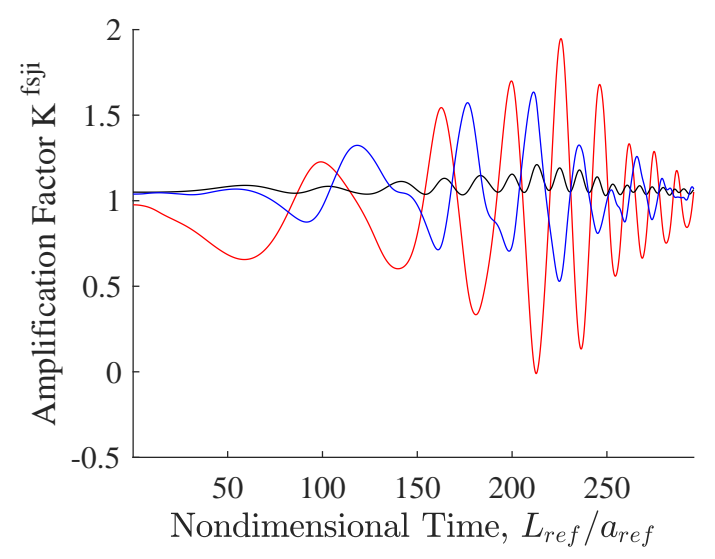

(c)

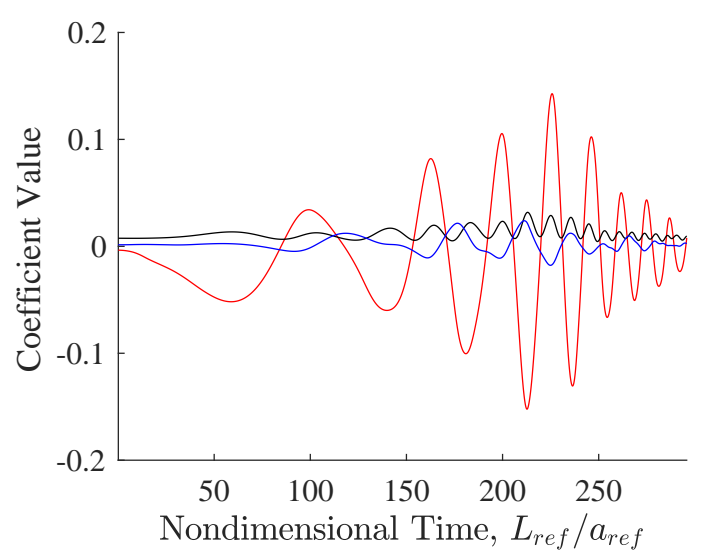

(b) Forced Pitch Response

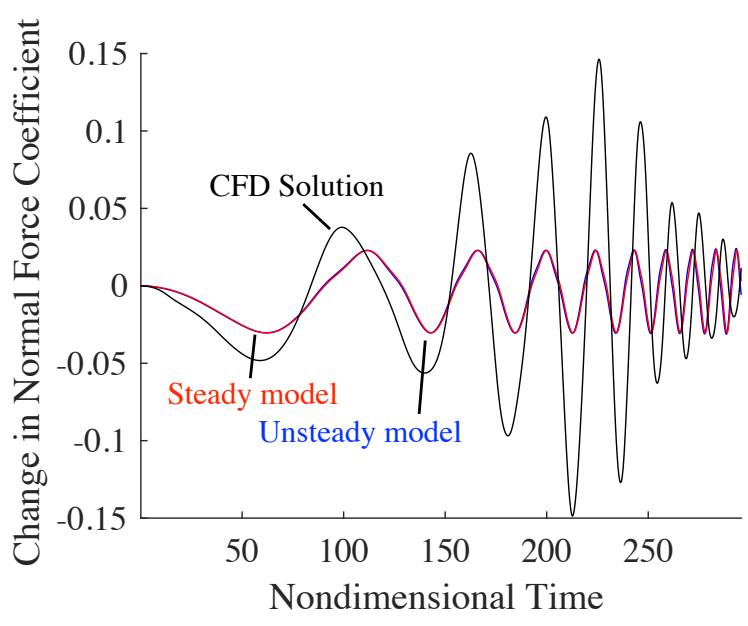

(d) Surrogate Modeling

Figure 8. Jet Interaction Sensitivity to Rigid-Body Motion. Normal Force (red), Axial Force (black), and Pitch Moment (blue).

well with the CFD solution and are unable to capture the gain in amplitude due to the increase in frequency. This example demonstrates some limitations of the approach. First, the nonlinear steady-state solution is missing any dynamic information from the system. Second, the unsteady approximation via convolution is extracted from an aerodynamic-only CFD solution with a small perturbation that may not be exciting as much of the dynamics in the flow solution as needed. The jet interaction changes the dynamics of the system, which may require extracting step responses with varying levels of jet interaction to better approximate the response to rigid-body dynamics.

Structural deformation is prescribed as a function of time with increasing frequency of oscillation from $1 \mathrm{~Hz}$ to approximately $50 \mathrm{~Hz}$ at the flight conditions listed in Table 7 . The history of the input and corresponding loads are presented in addition to the amplification factors associated with the multi-physics interaction in Figure 9. As expected from the quasi-steady analysis, the moment amplification factor has the most variation. However, the bounds on the amplification factor increase with increasing vibration frequency. For a flexible vehicle with attitude jet control the input is generally very short duration impulses, which will initiate some structural vibration leading to a modified applied force.

Table 7. Structural Deformation Simulation Conditions

\begin{tabular}{cccc}
\hline \hline Altitude, $\mathrm{km}$ & Mach & $\left|\eta_{1}\right|$ & Jet Total Pressure, MPa \\
21.3 & 3.0 & 2.0 & 17.5 \\
\hline
\end{tabular}




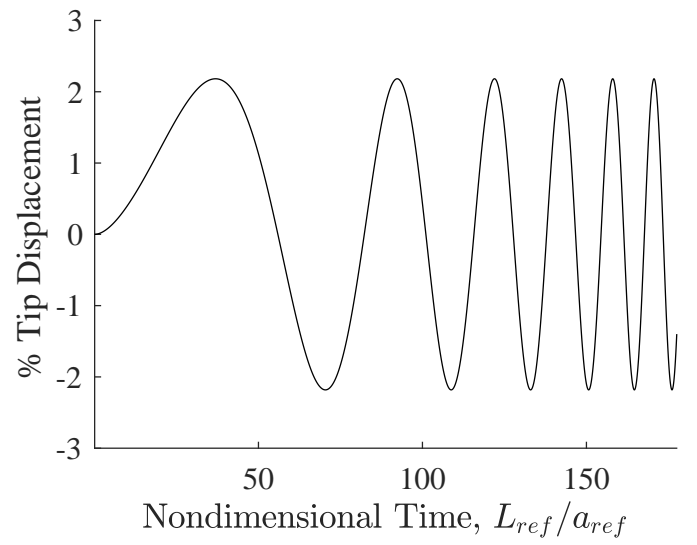

(a) Forced Deformation Input

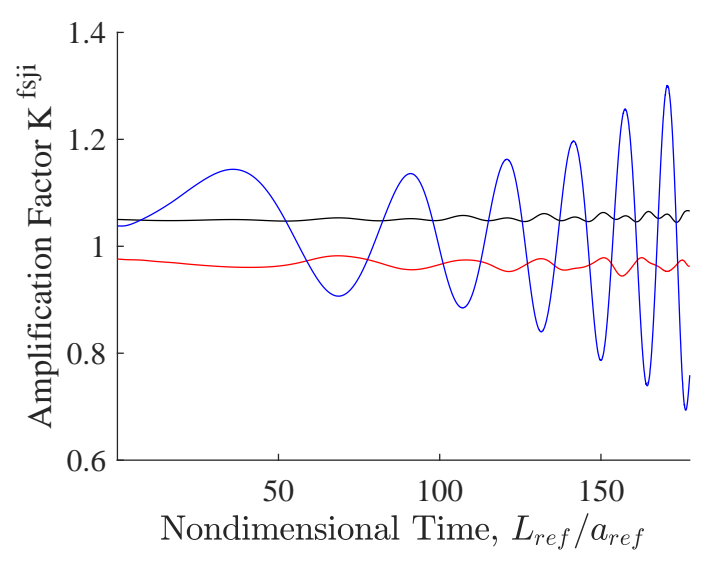

(c)

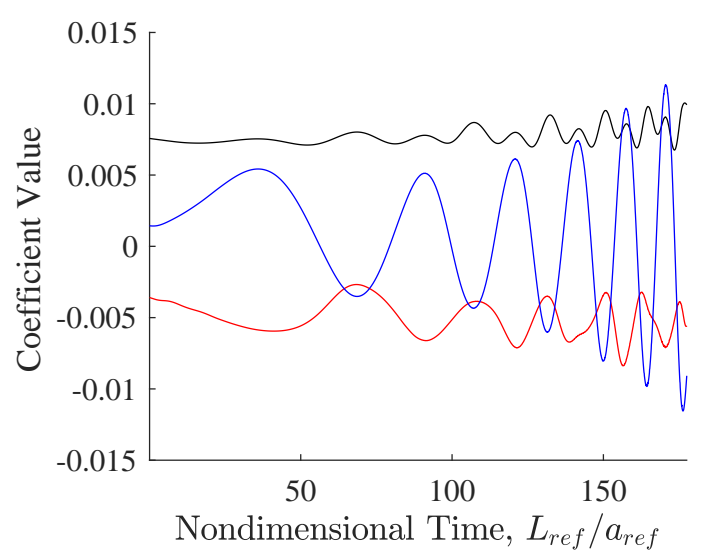

(b) Forced Deformation Response

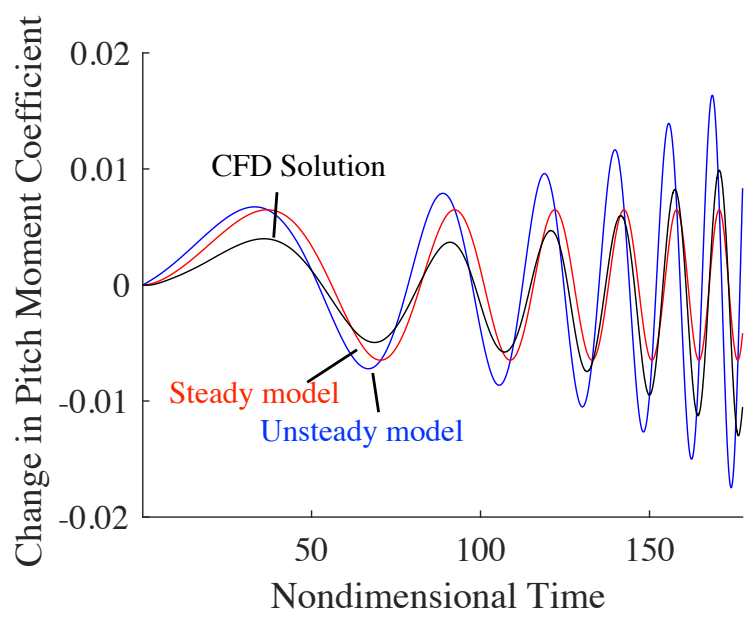

(d) Surrogate Modeling

Figure 9. Jet Interaction Sensitivity to Dynamic Structural Deformation. Normal Force (red), Axial Force (black), and Pitch Moment (blue).

Figure 9(d) presents the CFD solution along with the prediction of the unsteady model to highlight when flexibility and moderate deformation has a significant effect on the resultant loads. The unsteady model captures the frequency of the response well, but the amplitude is incorrectly modeled throughout the simulation. This effect is attributed to the jet interaction at these conditions changing the fluid dynamics significantly enough to not be captured using the step-response of the aerodynamic solution only to structural deformation. The current approach would need to be expanded to include the variation in the fluid dynamics to better approximate the applied loads.

The vehicle response to a step change in the jet total pressure is measured using a flexible vehicle. The step change is equivalent to $1 \%$ of the range for jet total pressure and the simulation begins with the jet in the "off" condition, which corresponds to a wall-type boundary condition. The boundary condition at the nozzle exit is then switched to a prescribed inflow condition equivalent to the jet exit conditions. The response of the vehicle loads and displacements is presented in Figure 10 along with the associated amplification factors. The simulation flight conditions are listed in Table 8.

Table 8. Dynamic Fluid-Structure-Jet Interaction Simulation Conditions

\begin{tabular}{ccccc}
\hline \hline Altitude, km & Mach & AoA, deg & Jet $(t=0)$ & Jet Total Pressure $(t>0), \mathrm{kPa}$ \\
21.3 & 3.0 & 0.0 & Off & 100 \\
\hline
\end{tabular}

The system shows an unstable response to the step in jet interaction pressure distribution with the stiffness 


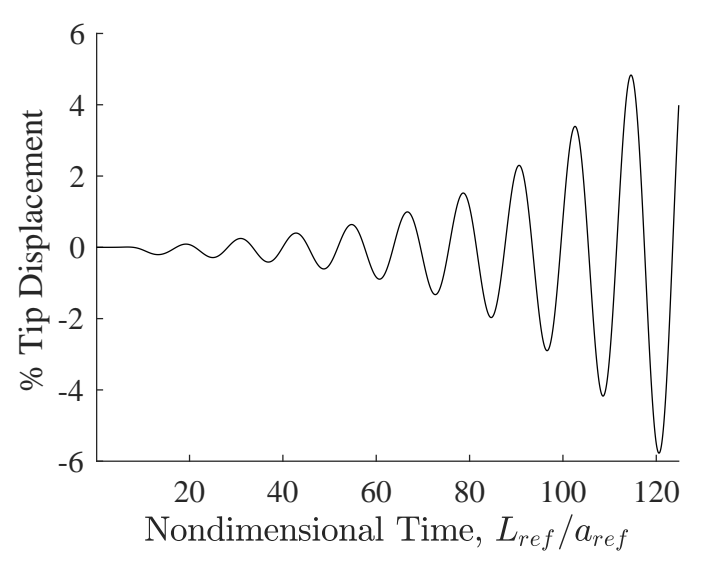

(a) Flexible Structure Response

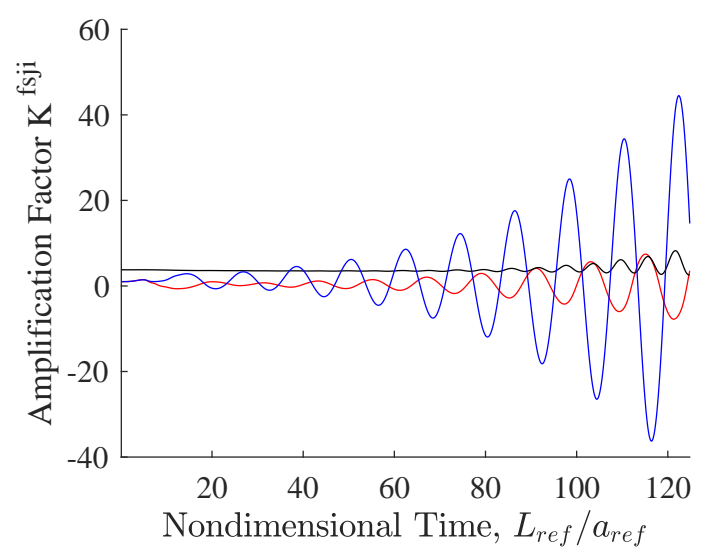

(c) FSJI Amplification Factor

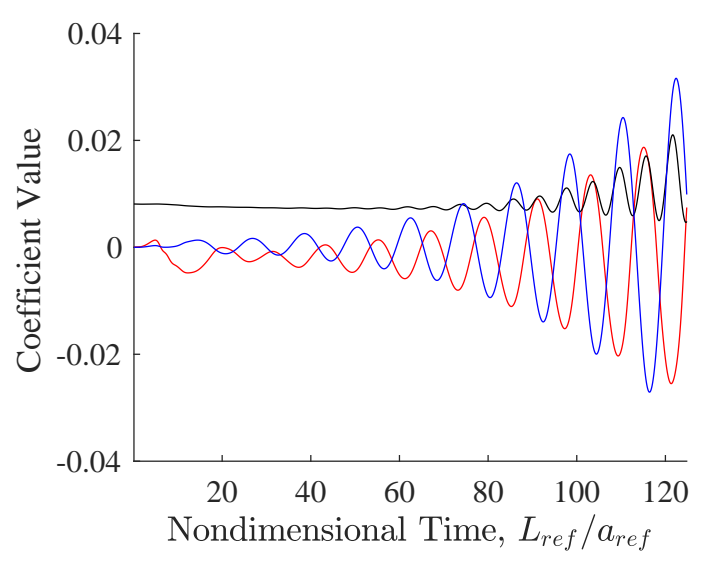

(b) Flexible Vehicle Response

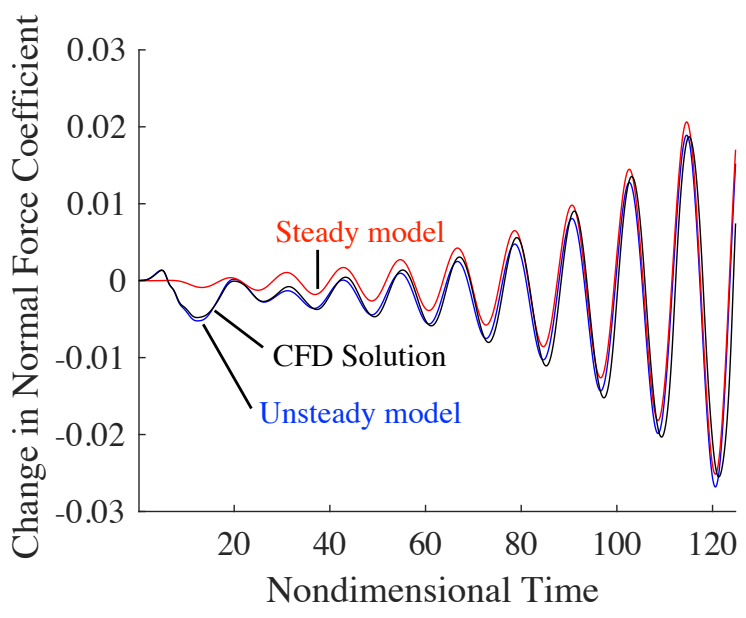

(d) Surrogate Modeling

Figure 10. Dynamic Fluid-Structure-Jet Interaction Simulation and Modeling. Normal Force (red), Axial Force (black), and Pitch Moment (blue).

values listed in Figure 2. This is not an accurate representation of the full system however, as the jet thrust is not accounted for in the CFD simulation due to the specification of the jet exit as an inflow boundary condition. In this setup only the aerodynamics and jet interaction contribute to the generalized forces. However, this case is still useful to assess how the jet interaction is affected by a flexible vehicle and how accurately the modeling approach is for this coupled simulation. Overall, the amplification factor response that includes the FSJI effects follows the same trends as the previous simulations. The unsteady model approximates the dynamics well for the flexible vehicle response and captures the jet transient component at the beginning of the solution. The unsteady approximation correlates well with the whole CFD solution throughout the simulation for each of the integrated force coefficient (see Figure 10(d)). This is a result of the minimal jet interaction induced by the small total pressure of the jet and can be approximated using the superposition of the jet response and deformation responses. This did not hold for the rigid-body and structural deformation simulations with 50 times the jet total pressure and a higher level of jet interaction. The current approach is therefore applicable to modeling unsteady loads with small levels of jet interaction, but will need to be enhanced to model stronger interactions between the jet and external flow.

\section{Concluding Remarks}

This paper presents the unsteady fluid-structure-jet interaction of a high-speed vehicle. This is an area of interest as design trends of increased slenderness and control jets are explored for highly maneuverable 
atmospheric flying vehicles. As the slenderness of the vehicle is increased, there is an increase in the structural flexibility leading to an interaction between the applied aerodynamic loads and structural dynamics. The control jets are included due to the known advantages over conventional control surfaces at high angles of incidence and high altitudes. The jet flow interacts with the external flow, which leads to a modified surface pressure distribution on the vehicle surface. For flexible vehicles this variation in pressure distribution has an effect on the structural dynamics. The highly-maneuverable application introduces very high angles of incidence and an unsteady operating condition that must be considered. This paper explores the effect of a dynamic environment on the fluid-structure-jet interaction problem, which had not been considered previously in the literature.

First, the jet sensitivity to large angles of attack and structural deformation is presented. A quasi-steady approach was used to expedite the CFD solution and achieve a finer resolution of the sensitivity to large angles of attack and deformation. The highly maneuverable vehicle operating conditions dictate a very wide range of flow conditions and structural deformations, which leads to studying angles of attack from 0 to 360 degrees and deformations up to $5 \%$ tip deflection. The results show that the jet interaction has a large effect when the jet is located on the windward side of the vehicle that leads to increases and decreases in the applied force and moment to the vehicle. The effect of jet interaction has a reversed effect at about 45 degrees angle of attack, which needs to be taken into account for aggressive high angle-of-attack maneuvers. The structural deformation has a small effect on the normal and axial force, but a significant effect on the vehicle pitch moment. In particular, the applied moment is amplified in the direction of the tip deformation, which can be exploited with control inputs to the jets that induce this type of deformation.

Second, the dynamic sensitivity of the problem due to step responses in the flow, structure, and jet are presented. These results are used to construct a linearized model of the system and compared to the nonlinear aeroelastic solution. This dynamic modeling method is a candidate for future modeling of the unsteady FSJI over the whole range of operating conditions. In addition, these results indicate that the transient jet interaction response time may be much longer than the aerodynamic response time. This effect was seen throughout the dynamic CFD simulations as the jet interaction forces tended to have a certain lag time especially in the normal force coefficient, which is dominated by the large expansion region that develops downstream of the nozzle exit.

Finally, time-accurate dynamic simulations of the vehicle with jet interaction and fluid-structure interaction were presented to study the effect of a dynamic environment and structural vibration to the FSJI problem. These unsteady simulations were compared against an unsteady modeling approach that was developed to highlight the nonlinear response and inform future modeling efforts of the dynamic FSJI over a full flight envelope. The unsteady model showed improved correlation with the CFD solution compared to the steady model and at worst replicated the steady-state approximation. A gain in the jet interaction response was observed for high-frequency rigid-body and structural motion, which was not captured in the models and may require additional step responses evaluated at different jet conditions. Overall, the unsteady modeling approach represents a feasible method to approximate the unsteady FSJI effects of high-speed vehicles, but further development is required to model cases with significant jet interaction.

Overall, this work adds to the jet interaction discussion in the literature and extends the previous FSJI work to the unsteady domain. The jet interaction sensitivity to a wide range of angles of attack and structural deformation was presented, which was required for understanding the highly maneuverable operating conditions of a flexible vehicle. A modeling method was adapted to the FSJI problem and has the potential to model the unsteady effects over a range of flight conditions. Dynamic aeroelastic solutions of the vehicle with transient jet interaction effects examines the intersection of the slenderness and control jet design trends and informs future high-speed vehicle designs.

\section{Acknowledgments}

This work was supported by the U.S. Air Force Research Laboratory Munitions Directorate with Dr. Crystal Pasiliao and Dr. Daniel Reasor as technical monitors. The CFD results were obtained primarily using the Lightning high performance computing system of the U.S. Air Force Research Laboratory DoD Supercomputing Resource Center. Opinions, interpretations, conclusions, and recommendations are those of the authors and are not necessarily endorsed by the U.S. Government. 


\section{References}

${ }^{1}$ Spaid, F. W. and Cassel, L. A., "Aerodynamic Interference Induced by Reaction Controls," Tech. Rep. 173, AGARD, December 1973.

${ }^{2}$ Spaid, F. W. and Zukoski, E. E., "A Study of the Interaction of Gaseous Jets from Transverse Slots with Supersonic External Flows," AIAA Journal, Vol. 6, No. 2, February 1968, pp. 205-212.

${ }^{3}$ Margason, R. J., "Fifty Years of Jet in Cross Flow Research," Computational and Experimental Assessment of Jets in Cross Flow, Advisory Group for Aerospace Research \& Development, 1993.

${ }^{4}$ HOAG, D. G., "The history of Apollo onboard guidance, navigation, and control," Journal of Guidance, Control, and Dynamics, Vol. 6, No. 1, January 1983, pp. 4-13.

${ }^{5}$ Hanna Prince, J. L., Chavis, Z. Q., and Wilmoth, R. G., "Modeling Reaction-Control-System Effects on Mars Odyssey," Journal of Spacecraft and Rockets, Vol. 42, No. 3, May 2005, pp. 444-449.

6 "NASA's Exploration Systems Architecture Study: Final Report," Tech. Rep. TM-2005-214062, NASA, 2005.

${ }^{7}$ Roger, R. P., "The Aerodynamics of Jet Thruster Control for Supersonic/Hypersonic Endo-Intercepters: Lessons Learned," 37th AIAA Aerospace Sciences Meeting and Exhibit, AIAA 99-0804, January 1999.

${ }^{8}$ Cassel, L. A., "Applying Jet Interaction Technology," Journal of Spacecraft and Rockets, Vol. 40, No. 4, July 2003, pp. 523-537.

$>{ }^{9}$ Randolph, H., Chew, L., and Johari, H., "Pulsed jets in supersonic crossflow," Journal of Propulsion and Power, Vol. 10, No. 5, September 1994, pp. 746-748.

${ }^{10}$ Naumann, K. W., Ende, H., George, A., and Mathieu, G., "Stationary and Time-Dependent Effects in the Near Interaction of Gaseous Jets and Supersonic Cross-flow," 29th AIAA Fluid Dynamics Conference, AIAA 1998-2972, 1998.

-11Ebrahimi, H. B., "Numerical Simulation of Transient Jet-Interaction Phenomenology in a Supersonic Freestream," Journal of Spacecraft and Rockets, Vol. 37, No. 6, November-December 2000, pp. 713-719.

$\checkmark 12$ Génin, F. and Menon, S., "Dynamics of sonic jet injection into supersonic crossflow," Journal of Turbulence, Vol. 11, 2010, pp. N4.

13 DeSpirito, J., "Turbulece Model Effects on Cold-Gas Lateral Jet Interaction in a Supersonic Crossflow," Journal of Spacecraft and Rockets, Vol. 52, No. 3, May-June 2015, pp. 836-852.

14 DeSpirito, J., "Transient Lateral Jet Interaction Effects on a Generic Fin-Stabilized Projectile," 30th AIAA Applied Aerodynamics Conference, AIAA 2012-2907, June 2012.

${ }^{15}$ Sahu, J., Fresconi, F., and Heavey, K. R., "Unsteady Aerodynamic Simulations of a Finned Projectile at a Supersonic Speed With Jet Interaction," Tech. Rep. ARL-TR-6960, U.S. Army Research Laboratory, Aberdeen Proving Ground, MD, June 2014.

${ }^{16}$ Kitson, R. C. and Cesnik, C. E. S., "Aeroelastic Modeling and Simulation of High-Speed Flexible Vehicles," 15th Dynamics Specialists Conference, AIAA 2016-1324, January 2016.

${ }^{17}$ Kitson, R. C. and Cesnik, C. E. S., "High Speed Vehicle Fluid-Structure-Jet Interaction Analysis and Modeling," 58th AIAA/ASCE/AHS/ASC Structures, Structural Dynamics, and Materials Conference, AIAA 2017-0405, January 2017.

${ }^{18}$ Biedron, R. T., Carlson, J., Derlaga, J. M., Gnoffo, P. A., Hammond, D. P., Jones, W. T., Kleb, B., Lee-Rausch, E. M., Nielsen, E. J., Park, M. A., Rumsey, C. L., Thomas, J. L., and Wood, W. A., "FUN3D Manual 12.9," Tech. Rep. NASA-TM-2016-219012, NASA, 2016.

- ${ }^{19}$ DeSpirito, J., "Factors Affecting Reaction Jet Interaction Effects on Projectiles," 29th AIAA Applied Aerodynamics Conference, AIAA 2011-3031, June 2011.

${ }^{20}$ Miller, W. A., Medwell, P. R., Kim, M., and Doolan, C. J., "Computational Methodology for Investigating the Transient Interaction between a Reaction Control Jet and a Hypersonic Crossflow," 54th AIAA Aerospace Sciences Meeting, AIAA 2016-0343, January 2016.

${ }^{21}$ Raveh, D. E. and Mavris, D. N., "Reduced-Order Models Based on CFD Impulse and Step Responses," 42nd AIAA/ASME/ASCE/AHS/ASC Structures, Structural Dynamics, and Materials Conference, AIAA 01-1527, April 2001.

${ }^{22}$ Skujins, T. and Cesnik, C. E. S., "Reduced-Order Modeling of Unsteady Aerodynamics Acorss Multiple Mach Regimes," Journal of Aircraft, Vol. 51, No. 6, November-December 2014, pp. 1681-1704. 